\title{
SOLUCIÓN COMPUTACIONAL DE MODELOS BIOLÓGICOS DE FORMACIÓN DE PATRONES ESPACIO-TEMPORALES
}

\section{COMPUTATIONAL SOLUTION OF BIOLOGICAL MODELS OF SPATIAL-TEMPORAL PATTERN FORMATION}

\author{
Juan C. Vanegas A. ${ }^{1} \quad$ Nancy S. Landinez P. ${ }^{1} \quad$ Diego A. Garzón A. ${ }^{2}$ \\ Recibido 21 de diciembre de 2007, aceptado 16 de junio de 2009 \\ Received: December 21, 2007 Accepted: June 16, 2009
}

\begin{abstract}
RESUMEN
Diversos modelos matemáticos han sido utilizados para describir patrones espaciales y temporales presentes en la naturaleza, como la pigmentación de la piel de algunos peces y las rayas del tigre. Estos modelos matemáticos pueden ser implementados mediante diversas técnicas numéricas entre las que se destacan el método de diferencias finitas, los elementos finitos y los métodos espectrales. En este artículo se describe un método de implementación por elementos finitos de dos modelos de morfogénesis, un modelo de formación de patrones y un modelo de movimiento celular. Los resultados obtenidos son comparables con los reportados en otros trabajos usando el método de las diferencias finitas. Se concluye que la técnica utilizada es válida para implementar este tipo de problemas y se espera sea de utilidad en la formulación e implementación de modelos matemáticos biológicos complejos de crecimiento y desarrollo celular y tisular.
\end{abstract}

Palabras clave: Reacción-difusión, modelos biológicos, método de elementos finitos, métodos numéricos, biología matemática.

\begin{abstract}
Several mathematical models have been used to describe spatial-temporal patterns present in nature such as the skin pigmentation of some fishes and the tiger striped pattern. These mathematical models can be implemented using a wide set of numerical methods among which the finite differences method, the finite elements method and spectral methods are commonly used. In this work, a finite element method is described for the solution of two morphogenesis models, a patternformation model and a cell-movement model. The results obtained are similar to those reported by other authors using different numerical approaches. This fact proves the technique to be suitable in the solution of these models and supports our expectations for its use in the solution of complex biological models of growth and cell and tissue development.
\end{abstract}

Keywords: Reaction-diffusion, biological models, finite elements method, numerical methods, mathematical biology.

\section{INTRODUCCIÓN}

La aplicación del modelamiento matemático a problemas de biología del desarrollo ha dado lugar a la formulación de una variedad de modelos que describen fenómenos de formación de patrones espacio-temporales [1]. Una clasificación de estos patrones desde el punto de vista biológico da lugar a dos categorías: patrones químicos y patrones de movimiento celular [2]. A su vez, en la categoría de patrones químicos existen dos tipos de modelos: modelos de gradiente y modelos de reacción-difusión.

Los modelos químicos de gradiente son aquellos que generan patrones a partir de sustancias químicas que experimentan diferencias de concentración y que durante su evolución temporal tienden a un estado uniforme en

1 Maestría en Ingeniería Biomédica. Grupo de Modelado Matemático y Métodos Numéricos (GNUM). Universidad Nacional de Colombia. Bogotá, Colombia. Edificio 407, oficina 103A. E-mail: jcvanegasa@ieee.org; nslandinezp@unal.edu.co

2 Profesor Departamento de Ingeniería Mecánica y Mecatrónica. Grupo de Modelado Matemático y Métodos Numéricos (GNUM). Universidad Nacional de Colombia. Bogotá, Colombia. Edificio 407, oficina 103A. E-mail: dagarzona@unal.edu.co 
el espacio y en el tiempo. En los modelos de reaccióndifusión, las interacciones químicas generan patrones complejos en el espacio y/o el tiempo, debido a que se encuentran términos de transporte, síntesis y degradación que dependen de todas las sustancias químicas presentes en el dominio de análisis [2]. Por su parte, los modelos de movimiento celular involucran la formación de patrones debido a cambios de densidad celular, por agregación o repulsión entre las células, o por respuesta a sustancias químicas concretas [3].

Turing [5] en 1952 propuso el primer modelo de morfogénesis en el cual supone la existencia de cierto número de químicos, denominados morfogenes, que difunden e interactúan en un medio de células del mismo linaje. Demostró que un sistema de reacción-difusión con estas características, definido por un dominio espacial cerrado y extenso, y bajo los parámetros apropiados, evoluciona en un patrón espacial heterogéneo debido a pequeñas perturbaciones de las concentraciones químicas, fenómeno conocido como inestabilidad por difusión $[4,6]$. En esta nueva teoría, la difusión adopta un nuevo rol contrario a la concepción clásica de estabilidad ya que su acción torna el estado estacionario homogéneo en inestable [7]. Es decir, un sistema presenta inestabilidad por difusión (o inestabilidad de Turing) si el estado estacionario es estable a pequeñas perturbaciones en ausencia de difusión, pero inestable a pequeñas perturbaciones espaciales cuando la difusión está presente [7, 9]. Estas inestabilidades de Turing, a su vez, se caracterizan por presentar un estado temporal estable a medida que evoluciona en el tiempo, y patrones inestables en el espacio, adecuados para describir problemas de morfogénesis [2, 3, 7]. Los detalles sobre las inestabilidades de Turing y la formación de los patrones espacio-temporales son tratados en [9].

Otro tipo característico de respuesta de los sistemas de reacción-difusión son las ondas viajeras. Este tipo de soluciones describen, desde el punto de vista físico, procesos de transición de un equilibrio a otro, adaptándose a las propiedades del medio y generando un patrón de onda que se desplaza desde las condiciones iniciales [10].

Todos estos modelos han sido ampliamente estudiados en la biología del desarrollo debido a que explican la formación de tejidos y órganos, permiten cuantificar la evolución de las distintas variables implicadas en el proceso de morfogénesis, y permiten entender la formación de tejidos y órganos a partir de la formación de patrones espacio-temporales [4]. La evolución de estos modelos trae consigo el desarrollo de disciplinas asociadas y la formulación matemática de diversidad de procesos [2], como la descripción de la acción oscilatoria de ciertos pares químicos [11], la predicción de la formación de los patrones de pigmentación de la piel en varios animales [7, 8], la formación de patrones de crecimiento de los tallos de algunas plantas [12], modelos del crecimiento de tejidos como hueso y cartílago [13], y más recientemente, la formación de las ramas de la epidermis que dan lugar al espaciamiento de los folículos pilares [14]. La figura 1 ilustra un ejemplo de formación de patrones de pigmentación de la piel. Arriba, un jaguar cuya piel muestra anillos oscuros sobre un fondo más claro. Abajo, la obtención numérica de éstos anillos.
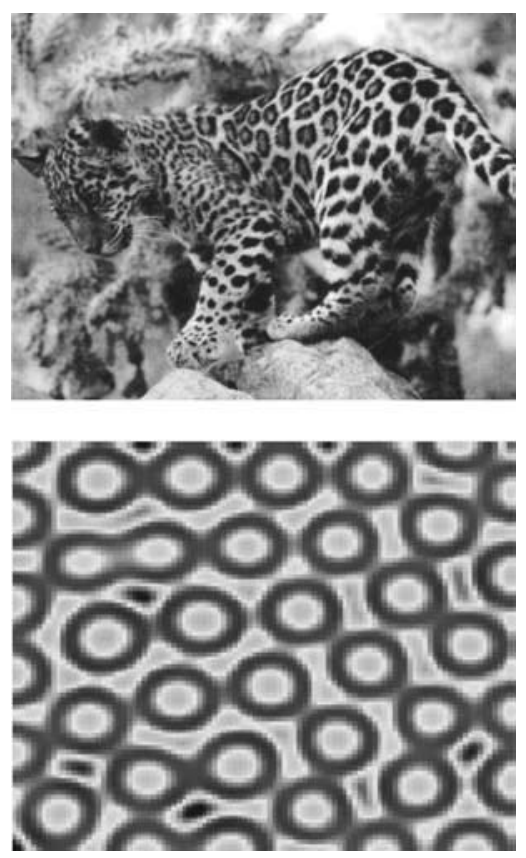

Figura 1. Ejemplo de un patrón de pigmentación de la piel [8].

Teniendo en cuenta la descripción general de los diferentes tipos de modelos biológicos, en el presente artículo se han considerado dos modelos químicos formulados a partir de ecuaciones de reacción-difusión cuya respuesta presenta inestabilidades de Turing, y un modelo de formación de onda viajera que describe un proceso de movimiento celular. El objetivo es realizar una implementación numérica de estos modelos utilizando el método de los elementos finitos y establecer criterios de comparación con la solución obtenida mediante otros métodos numéricos como las diferencias finitas, reportados en [1-2, 4, 6, 8]. En la siguiente sección se hace una breve explicación de las ecuaciones de reacción-difusión y a continuación se presentan los modelos biológicos utilizados. El método de los elementos finitos mediante el cual estos modelos son implementados es presentado en la sección "Solución por el método de los elementos finitos" y en la sección 
de "Resultados" se ilustran las soluciones numéricas obtenidas. Finalmente, se discuten los resultados.

\section{ECUACIONES DE REACCIÓN-DIFUSIÓN}

Un problema de difusión modela el movimiento de una densidad de individuos en un entorno cualquiera $[1,2$, $7,12]$. Los individuos pueden ser tan pequeños como partículas físicas fundamentales, moléculas, células o bacterias, e inclusive pueden ser organismos más complejos como animales, plantas, personas o fenómenos de distribución como una epidemia [1, 2, 8]. Si todos los individuos residen en una región, se puede decir que la concentración de individuos está limitada por una región $S$ del espacio que define un volumen $\mathrm{V}$, contenido dentro de un dominio espacial $\Omega$ en el interior del cual se concentra el movimiento. En el caso de una única especie, sea $u(\mathrm{t}, \mathbf{x})$ la densidad de población, donde $t$ es el tiempo y $\mathbf{x}$ la variable espacial. Para conocer cómo varía la población total conforme a la variación en el espacio $\mathbf{x}$ y la evolución en el tiempo $t$ del total de los individuos, se puede determinar el movimiento de $u(t, \boldsymbol{x})$, o término difusivo, denotado por un vector que apunta hacia la dirección de mayor decrecimiento denominado vector de flujo de densidad de población, esto es, la población fluye desde puntos de mayor concentración hacia puntos de menor concentración $[15,16]$. Este principio es conocido como Ley de Fick y se expresa de la siguiente forma (1):

$$
\mathbf{J}(t, \mathbf{x})=-D \nabla u(t, \mathbf{x})
$$

donde $\mathbf{J}(\mathrm{t}, \mathbf{x})$ es el vector de flujo de $u(t, \boldsymbol{x})$, y $D$ es el coeficiente de difusión.

A su vez, la población de individuos puede verse modificada por procesos internos como nacimiento, muerte, o reacciones químicas, que establecen un término reactivo adicional en la función de densidad poblacional $u(t, \boldsymbol{x})$ denotado por $f(t, \boldsymbol{x}, u)$. De acuerdo al principio de conservación, la razón de cambio de la cantidad de materia contenida en $V$ debe ser igual al flujo neto de materia a través de la superficie $S$ que la delimita, más la cantidad de materia transformada al interior de $V$ debido al término reactivo. Esto expresado matemáticamente es (2):

$$
\frac{\partial}{\partial t} \int_{V} u(t, \mathbf{x}) d V=-\int_{S}(\mathbf{J} \cdot \overline{\mathbf{n}}) d s+\int_{V} f d V
$$

donde $\bar{n}$ es el vector normal a la superficie $S$. Utilizando el teorema de la divergencia en el término difusivo y combinando (1) y (2) se obtiene (3):

$$
\frac{\partial}{\partial t} \int_{V} u(t, \mathbf{x}) d V=\int_{V} \nabla \cdot(D \nabla u(t, \mathbf{x})) d V+\int_{V} f d V
$$

Esta nueva ecuación corresponde a una ecuación diferencial definida en el dominio $\Omega=V$ con unas condiciones de contorno definidas por la superficie $\Gamma=S$ que rodea al volumen $V$. Expresando (3) en forma diferencial se obtiene (4):

$$
\underbrace{\frac{\partial u}{\partial t}}_{\text {TASA DE CAMBIO }}=\underbrace{\nabla \cdot(D \nabla u)}_{\text {T. DIFUSIVO }}+\underbrace{f}_{\text {T. REACTIVO }}
$$

La ecuación (4) se conoce como ecuación de reaccióndifusión y permite, junto con las condiciones de contorno dadas, predecir la evolución de los individuos de la especie denotada por $u(t, \boldsymbol{x})$. El análisis anterior es válido para un sistema de una única especie de individuos. Sin embargo, el resultado en (4) puede extenderse a varias especies de individuos denotando $u(t, \boldsymbol{x})$ como $\boldsymbol{u}(t, \boldsymbol{x})$ o vector de densidad de población.

\section{MODELOS IMPLEMENTADOS}

Existen diferentes modelos biológicos que permiten obtener una descripción matemática de fenómenos complejos presentes en la naturaleza [1-4, 7, 12]. Como se mencionó en la sección 2, estos modelos se pueden clasificar en modelos de formación de patrones y modelos de movimiento celular [2,3]. En el conjunto de los modelos de formación de patrones tres modelos están bien referenciados: el modelo fenomenológico de Gierer-Meinhardt [2, 4], modelo de morfogénesis de Schnakenberg [1-2, 4, 7-8], y el modelo de glucólisis $[2,7,8]$, utilizado para explicar la relación activador-inhibidor entre sustancias químicas. Estos modelos generan patrones espaciales y cumplen con los criterios de estabilidad de Turing analizados en [9]. Un cuarto modelo típicamente utilizado para ilustrar el movimiento celular, consecuencia de la interacción química con el entorno, es el modelo de quimiotaxis [8]. Este modelo genera patrones espacio-temporales ya que su solución tiene la forma de una onda viajera. Una descripción detallada de cada uno de estos modelos es presentada a continuación.

\section{Modelo de Gierer-Meinhardt}

El modelo de Gierer-Meinhardt es un modelo fenomenológico de reacción cinética en el que una de las sustancias químicas, el activador, inicia la producción de la segunda sustancia, el inhibidor, que a su vez detiene la producción 
del activador. En su forma adimensional, el modelo está dado por las siguientes ecuaciones $(5)[6,15,17]$ :

$$
\begin{aligned}
& \underbrace{\frac{\partial u}{\partial t}}_{1}=\underbrace{\nabla^{2} u}_{2}+\gamma(\underbrace{a}_{3}-\underbrace{b u}_{4}+\underbrace{\frac{u^{2}}{v\left(1+k u^{2}\right)}}_{5}) \\
& \underbrace{\frac{\partial v}{\partial t}}_{1}=\underbrace{d \nabla^{2} v}_{2}+\gamma(\underbrace{u^{2}-\underbrace{v}_{4}}_{3})
\end{aligned}
$$

En (5) $u$ es la sustancia activadora y $v$ es la sustancia inhibidora. Las constantes $a, b, d$ y $\gamma$ son todas parámetros positivos adimensionales y $k$ es una medida de la concentración de saturación. El término 1 representa flujo, 2 representa difusión, 3 representa producción, 4 representa consumo. En (5a), el término 5 representa tanto autocatálisis en $u$ con saturación para altos valores de concentración, como inhibición de $u$ mediante la producción de $v$ [6].

\section{Modelo de Schnakenberg}

El modelo de Schnakenberg es ampliamente difundido por ser uno de los modelos de reacción-difusión más sencillo y utilizado en morfogénesis [1, 2]. Este modelo determina el comportamiento de un químico activador $u$ en presencia de un químico inhibidor $v$. En su forma adimensional, el modelo está descrito por (6):

$$
\begin{aligned}
& \underbrace{\frac{\partial u}{\partial t}}_{1}=\gamma(\underbrace{a}_{2}-\underbrace{u}_{3}+\underbrace{u^{2} v}_{4})+\underbrace{\nabla^{2} u}_{5} \\
& \underbrace{\frac{\partial v}{\partial t}}_{1}=\gamma(\underbrace{b-\underbrace{u^{2} v}_{4}}_{2})+\underbrace{d \nabla^{2} v}_{5}
\end{aligned}
$$

En la ecuación (6) el término 1 representa flujo, 2 representa producción, 3 representa consumo, 4 representa catálisis no lineal, y 5 representa difusión. Las constantes $a, b, d$ y $\gamma$ son todas parámetros positivos, siendo $a$ y $b$ valores adimensionales de producción, $\gamma$ una constante adimensional y $d$ un valor de difusión [2, 6]. La reacción cinética es tal que en la ecuación (6a) el término 4 representa la producción de $u$ en presencia de $v$, en tanto que en la ecuación (6b) el mismo término representa el consumo de $v$ en presencia de $u$. El modelo se utiliza como base matemática para análisis de estabilidad y formación de patrones $[1,4]$, en la predicción de la interacción entre sistemas químicos moleculares $[2,11]$ y en la morfogénesis de formación y crecimiento de hueso [2].

\section{Modelo de Glucólisis}

Un segundo modelo que predice la formación de patrones de origen biológico es el modelo de glucólisis. La glucólisis o glicólisis es el proceso de síntesis de la molécula de glucosa para proporcionar energía al metabolismo celular. A través de una secuencia de reacciones, la glucosa es transformada en piruvato y en ATP, unidad de intercambio metabólico en el organismo vivo [8]. En su forma adimensional, el modelo está dado por las ecuaciones (7):

$$
\begin{aligned}
& \underbrace{\frac{\partial u}{\partial t}}_{1}=\underbrace{D_{u} \nabla^{2} u}_{2}+\underbrace{\delta}_{3}-\underbrace{k u}_{4}-\underbrace{u^{2} v}_{5} \\
& \frac{\partial v}{\partial t}=\underbrace{D_{v} \nabla^{2} v}_{2}+\underbrace{k u}_{3}+\underbrace{u v^{2}}_{6}-\underbrace{v}_{7}
\end{aligned}
$$

En la ecuación (7) el término 1 representa flujo, 2 representa difusión, 3 representa producción, 4 representa degradación, 5 representa consumo no lineal, 6 representa activación no lineal, y 7 representa consumo. La interpretación biológica es similar al modelo de Schnakenberg, siendo $u$ la concentración de glucosa, $v$ la producción de piruvato, $D_{u}$ y $D_{v}$ los coeficientes de difusión, el término $u^{2} v$ representando consumo no lineal de $u$ y el término $u v^{2}$ representando la activación no lineal de $v$. $\delta$ es un parámetro positivo que representa la constante de formación de glucosa. El parámetro $k$, también positivo, representa en $u$ el consumo natural de glucosa, mientras que en $v$ representa la producción, en la misma proporción, de piruvato. Este modelo representa la heterogeneidad de un tejido para la transformación de glucosa en piruvato simulando la realidad del fenómeno $[2,8]$.

\section{Modelo de Quimiotaxis}

El cuarto modelo corresponde a un modelo de movimiento celular que describe la formación de una onda viajera en virtud de un químico o quimiotaxis. Aunque este modelo no produce específicamente patrones espacio-temporales, sí proporciona una solución que bien describe el fenómeno biológico, variante en el tiempo y en el espacio. La quimiotaxis es el fenómeno de migración celular en el cual las células dirigen su movimiento de acuerdo a gradientes de concentración en ciertos químicos presentes en el entorno celular denominados quimioatractantes [18]. De acuerdo con Painter [8], el modelo de quimiotaxis más simple consiste en una población celular que responde a gradientes de quimioatractante. Un primer acercamiento al modelo desprecia el crecimiento celular, esto es, 
considera que el movimiento celular se lleva a cabo más rápidamente que la división celular. El modelo con esta condición está descrito por (8):

$$
\begin{aligned}
& \underbrace{\frac{\partial n}{\partial t}}_{1}=\nabla \cdot[\underbrace{D_{n} \nabla n}_{2}-\underbrace{n \alpha \Delta u}_{3}] \\
& \frac{\partial u}{\partial t}=\underbrace{D_{u} \nabla^{2} u}_{2}+\underbrace{g(n)}_{4}-\underbrace{\beta n u}_{5}-\underbrace{\delta u}_{6}
\end{aligned}
$$

En (8) $n$ es la densidad celular, $u$ es la concentración de quimioatractante, $D_{u}$ y $D_{v}$ son los coeficientes de difusión, $\alpha$ es la sensibilidad al quimioatractante, $\beta$ es la tasa de degradación química celular, $\delta$ es la tasa de degradación natural celular, y $g(n)$ es una función de la producción de quimioatractante por parte de las células. En (7), el término 1 representa flujo, 2 representa difusión, 3 representa quimiotaxis, 4 representa producción dependiente, 5 representa consumo, y 6 representa degradación.

Teniendo en cuenta que el desplazamiento celular puede ocurrir conjuntamente con procesos de división mitótica y pérdida celular, situación más semejante a la realidad biológica, el modelo sufre algunas alteraciones y es descrito por (9) [8]:

$$
\frac{\partial n}{\partial t}=\nabla \cdot\left[D_{n} \nabla n-n \alpha \nabla u\right]+r \underbrace{f(u) n\left(1-\frac{n}{n_{o}}\right)}_{1}-\delta_{2}^{\underbrace{}_{n} n}
$$

$\frac{\partial u}{\partial t}=D_{u} \nabla^{2} u+g(n)-\delta_{u} u$

En (9) los parámetros se corresponden con los del modelo anterior. El término 1 en la ecuación de $n$ corresponde a una variación del modelo logístico para considerar la mitosis. El término 2 representa pérdida por consumo. $\delta_{n}$ corresponde a pérdida celular por migración hacia zonas distintas a las demarcadas por la acción del quimioatractante. En la siguiente sección se presenta la técnica de solución numérica utilizada para implementar los modelos anteriores mediante el método de los elementos finitos.

\section{SOLUCIÓN POR EL MÉTODO DE LOS ELEMENTOS FINITOS}

El método de los elementos finitos es un método numérico para resolver problemas complejos que utiliza como principio la simplificación y subdivisión del problema [19-22]. Si se divide el dominio de solución del problema en pequeñas unidades discretas no intersectantes llamadas elementos, compuestos de puntos extremos denominados nodos, y se obtiene la respuesta al problema en cada elemento, es posible construir una respuesta general a partir del conocimiento de las respuestas de todos los elementos $[19,20]$. El método que se presenta se implementa mediante una rutina de usuario programada en Fortran, de forma particular para cada modelo, cuyo fundamento matemático se encuentra en el método de ponderación de los residuos [19-21].

Sea un dominio $\Omega$ divido en cierto número $N E$ de elementos, cada uno definido en un subdominio $\Omega^{e}$ de $\Omega$, como muestra la figura 2 .

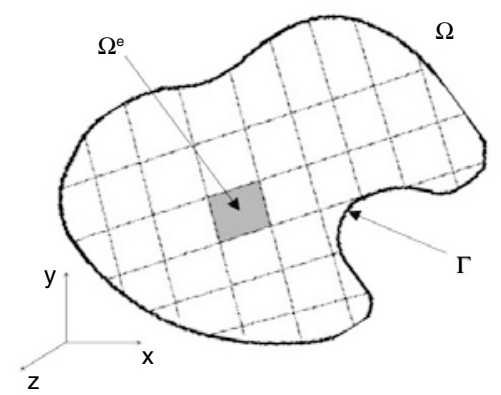

Figura 2. División del dominio $\Omega$ en $N E$ subdominios $\Omega^{e}$.

Para cada $\Omega^{e}$ existe un residuo $R^{e}$ o error entre la solución numérica local y la solución exacta. Si se toma la suma de los residuos locales, es posible obtener un residuo global $R^{g}$ y una solución global del problema definido en el dominio $\Omega$. Para el caso unidimensional de la ecuación de reacción-difusión expresada en (4), se quiere que el error en el dominio $\Omega$ cumpla con la relación (10):

$$
\int_{\Omega} \mathrm{R}^{\mathrm{g}} w \cdot d x=0
$$

donde $R^{g}$ es el residuo en $\Omega$ definido como (11):

$$
\mathrm{R}^{\mathrm{g}}=\frac{\partial u}{\partial t}-D \frac{\partial^{2} u}{\partial x^{2}}-f
$$


En (11) $u:=u(t, x), f:=f(t, x, u)$ y $w$ es una función de ponderación [19]. A partir de esta formulación es posible obtener (12) como la forma residual ponderada de la ecuación (4):

$$
\int_{\Omega}\left(\frac{\partial u}{\partial t}-D \frac{\partial^{2} u}{\partial x^{2}}-f\right) w \cdot d x=0
$$

Resolviendo la integral para cada uno de los términos, y haciendo integración por partes en el segundo término, se obtiene (13):

$$
\begin{aligned}
& \int_{\Omega} \frac{\partial u}{\partial t} w d x+\left[D \frac{\partial u}{\partial x} w\right]_{\Gamma} \\
& +\int_{\Omega} D \frac{\partial u}{\partial x} \frac{\partial w}{\partial x} d x-\int_{\Omega} f w \cdot d x=0
\end{aligned}
$$

En (13) $\Gamma$ es el contorno del dominio $\Omega$. La evaluación de (13) en cada subdominio $\Omega^{e}$ o elemento da lugar a la expresión (14):

$$
\int_{\Omega^{e}} \frac{\partial u}{\partial t} w d x+\int_{\Omega^{e}} D \frac{\partial u}{\partial x} \frac{\partial w}{\partial x} d x-\int_{\Omega^{e}} f w d x=0
$$

La solución de (14) requiere la introducción del concepto de funciones de forma. Las funciones de forma son funciones de interpolación definidas para cada elemento y constituyen la base para la construcción de una solución continua [19]. Para el caso lineal y unidimensional de un problema con elementos de dos nodos, las funciones de forma normalizadas, definidas en el intervalo $-1 \leq \xi \leq 1$ se expresan como (15) [22]:

$$
\begin{aligned}
& N_{1}(\xi)=\frac{1}{2} \xi(1-\xi) \\
& N_{2}(\xi)=\frac{1}{2} \xi(1+\xi)
\end{aligned}
$$

La figura 3 muestra una representación gráfica de estas funciones de forma, donde los nodos corresponden, en el dominio $\Gamma$ del elemento, a los puntos 1 y 2 , mientras que en el dominio general $\Omega$ los mismos nodos son denotados como $i$ e $i+l$ ya que pueden corresponder a los nodos de elementos adyacentes. La solución de (15) es válida en un único elemento, razón por la cual es necesario aplicar el mismo procedimiento a todos los elementos en $\Omega$.

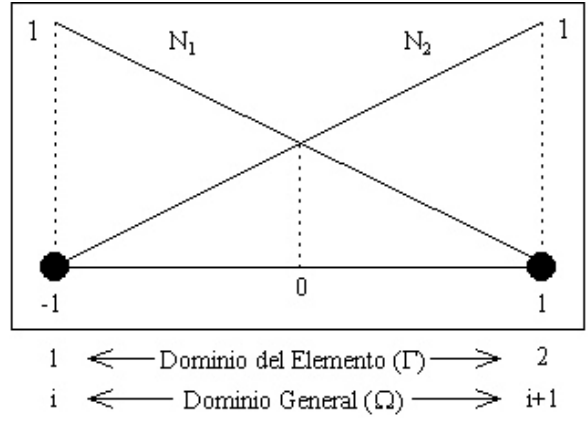

Figura 3. Representación gráfica de las funciones de forma.

Si $u_{1}$ y $u_{2}$ son los valores de $u(t, x)$ en los nodos 1 y 2 de un elemento cualquiera, la variación lineal o interpolación entre estos dos valores a lo largo de dicho elemento está dada por (16):

$$
u(\xi)=\left[\begin{array}{ll}
N_{1} & N_{2}
\end{array}\right] \cdot\left[\begin{array}{l}
u_{1} \\
u_{2}
\end{array}\right]
$$

Definiendo $x$ en términos de la base establecida por las funciones de forma, esto es, expresando $x$ como una interpolación de los valores nodales de $x$ para un elemento con nodos en las coordenadas $x_{1}$ y $x_{2}$ se obtiene (17):

$$
x(\xi)=\left[\begin{array}{ll}
N_{1} & N_{2}
\end{array}\right] \cdot\left[\begin{array}{l}
x_{1} \\
x_{2}
\end{array}\right]
$$

Esta parametrización establece la relación entre el dominio matemático normalizado $-1 \leq \xi \leq 1$ y el espacio físico $x_{1} \leq x \leq x_{2}$. Por su parte, la función de ponderación escogida es de la forma (18):

$$
w=\left[\begin{array}{l}
N_{1} \\
N_{2}
\end{array}\right]
$$

La escogencia de las funciones de ponderación como en (18) se conoce como el método de Galerkin [19, 20]. Si se cambia el espacio de integración de $x$ a $\xi$ mediante el jacobiano de la transformación, expresado como la derivada de $x(\xi)$, y se ajusta la notación vectorial, la expresión (14) puede reescribirse como (19): 


$$
\begin{aligned}
& \left(\int_{-1}^{1}\left(\frac{1}{\Delta t}\left[\begin{array}{c}
N_{1} \\
N_{2}
\end{array}\right] \cdot\left[\begin{array}{ll}
N_{1} & N_{2}
\end{array}\right] \cdot \frac{1}{J}\right) d \xi\right) \cdot\left[\begin{array}{l}
u_{1} \\
u_{2}
\end{array}\right] \\
& \left.+\left(\int_{-1}^{1}\left[\begin{array}{c}
\frac{\partial N_{1}}{\partial \xi} \\
\frac{\partial N_{2}}{\partial \xi}
\end{array}\right] \cdot\left[\begin{array}{ll}
\frac{\partial N_{1}}{\partial \xi} & \frac{\partial N_{2}}{\partial \xi}
\end{array}\right] \cdot \frac{1}{J}\right] d \xi\right] \cdot\left[\begin{array}{l}
u_{1} \\
u_{2}
\end{array}\right] \\
& -\left(\int_{-1}^{1}\left(f(t, x(\xi), u(t, x(\xi)))\left[\begin{array}{c}
N_{1} \\
N_{2}
\end{array}\right] \cdot \frac{1}{J}\right) d \xi\right)=0
\end{aligned}
$$

En (19) la función $f$ es el término reactivo de la expresión (4). La expresión (19) puede reducirse a un sistema matricial de tipo elemental expresado como (20):

$$
k \cdot u=f
$$

La expresión (20) corresponde a la discretización algebraica en el dominio $\Omega^{e}$ de un elemento, siendo $k$ la matriz de rigidez elemental, $u$ el vector elemental de incógnitas y $f$ el vector elemental de entradas. Ensamblando el resultado de (19) para el total $N E$ de elementos en $\Omega$ [19, $20,21]$ se obtiene un sistema matricial general definido como (21):

$$
\mathbf{K} \cdot \mathbf{U}=\mathbf{F}
$$

En (21) $\mathbf{K}$ es la matriz global de rigidez, $\mathbf{U}$ el vector de incógnitas y $\mathbf{F}$ el vector global de entradas. Este método matemático es aplicable a problemas de mayor dimensión. Una formulación para problemas bidimensionales y tridimensionales puede encontrarse en [19] y [20]. Para el caso de problemas bidimensionales, las funciones de forma normalizadas aumentan en número de acuerdo al número de nodos del elemento bidimensional. Si el elemento que se selecciona es un cuadrilátero, las funciones de forma se expresan como (22) [22]:

$$
\begin{aligned}
& N_{1}(\xi)=\frac{1}{4}\left(1-\xi_{1}\right)\left(1-\xi_{2}\right) \\
& N_{2}(\xi)=\frac{1}{4}\left(1-\xi_{1}\right)\left(1+\xi_{2}\right) \\
& N_{3}(\xi)=\frac{1}{4}\left(1+\xi_{1}\right)\left(1-\xi_{2}\right) \\
& N_{4}(\xi)=\frac{1}{4}\left(1+\xi_{1}\right)\left(1+\xi_{2}\right)
\end{aligned}
$$

\section{RESULTADOS}

Las soluciones numéricas presentadas han sido obtenidas utilizando la metodología desarrollada en la sección 4 y el software antes mencionado. El hardware utilizado es un PC de escritorio con procesador AMD Athlon 64 de $2.4 \mathrm{GHz}$ y $1 \mathrm{~GB}$ de memoria RAM.

\section{Modelo de Gierer-Meinhardt}

El modelo descrito por las ecuaciones (5) fue implementado en el dominio unidimensional [0,1] utilizando 300 elementos cuadráticos lagrangianos y 601 nodos. La figura 4 muestra los resultados para cuatro diferentes modos de vibración de la solución. En todos los casos a = 0.1, b = 1.0 y d = 72 [17]. Las condiciones de flujo en el contorno se consideran iguales a cero (flujo nulo). La condición inicial corresponde a una perturbación del 5\% alrededor del estado temporal estable. Se usaron 100 iteraciones con pasos de tiempo $\Delta \mathrm{t}=0.01$ en todas las simulaciones.
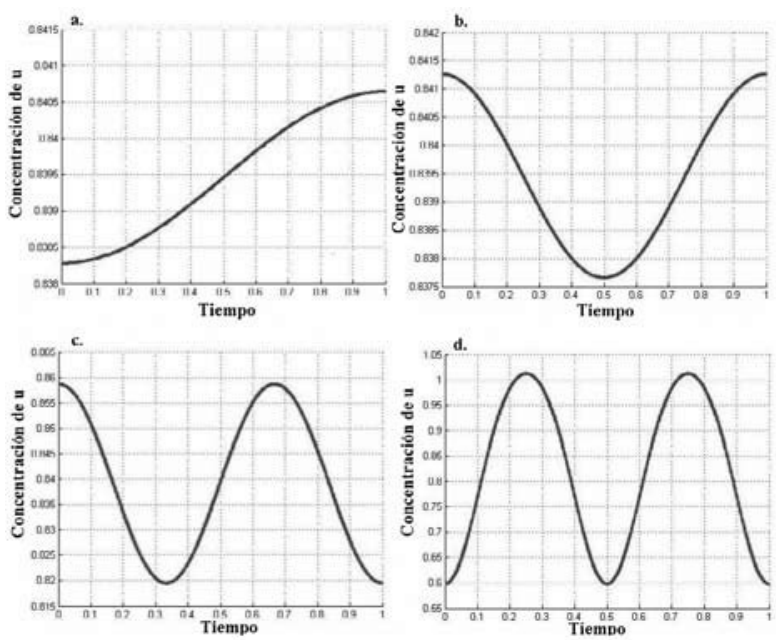

Figura 4. Solución del modelo de Gierer-Meinhardt. a) $\gamma=70$, b) $\gamma=270$, c) $\gamma=650$, d) $\gamma=1.100$.

Los resultados muestran la formación del patrón espacial en cuatro diferentes modos de vibración de acuerdo con los parámetros seleccionados [9, 17]. Estas variaciones del patrón demuestran la dependencia del modelo con respecto a sus parámetros [17]. Los resultados obtenidos concuerdan con los reportados por otros autores $[6,17]$.

\section{Modelo de Schnakenberg}

Los resultados para el modelo descrito por las ecuaciones (6) en el dominio unidimensional $[0,1]$ se muestran en las figuras 5 y 6 . En la implementación se usaron los siguientes parámetros: $\mathrm{a}=0.1, \mathrm{~b}=0.9, \mathrm{~d}=10, \gamma=789[2,17]$. Las 
condiciones de flujo en el contorno se consideran iguales a cero (flujo nulo). Se realizan 1.000 iteraciones, con un paso de tiempo $\Delta \mathrm{t}=0.005$, se utilizan 300 elementos cuadráticos lagrangianos y 601 nodos. Las flechas en la figura señalan la dirección hacia la cual evoluciona la respuesta hasta alcanzar el estado espacial estable. La condición inicial corresponde a una perturbación del 5\% alrededor del estado temporal estable.

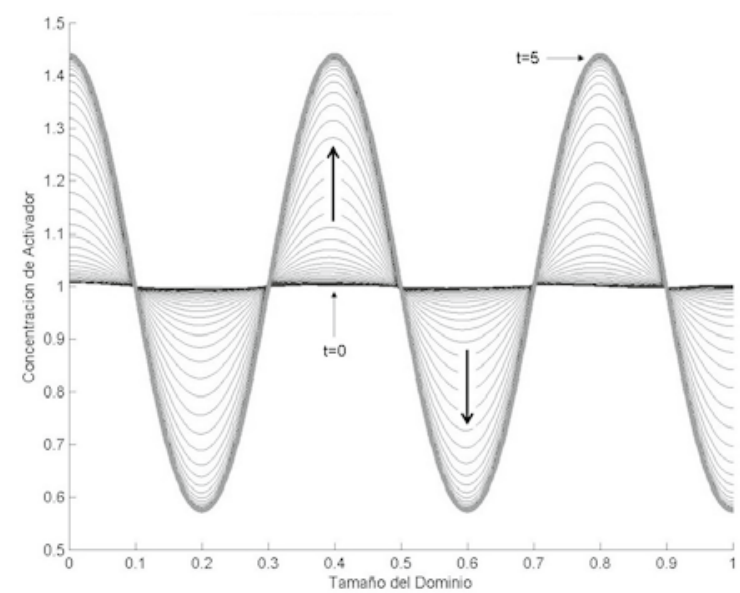

Figura 5. Solución del modelo de Schnakenberg. Concentración de químico activador.

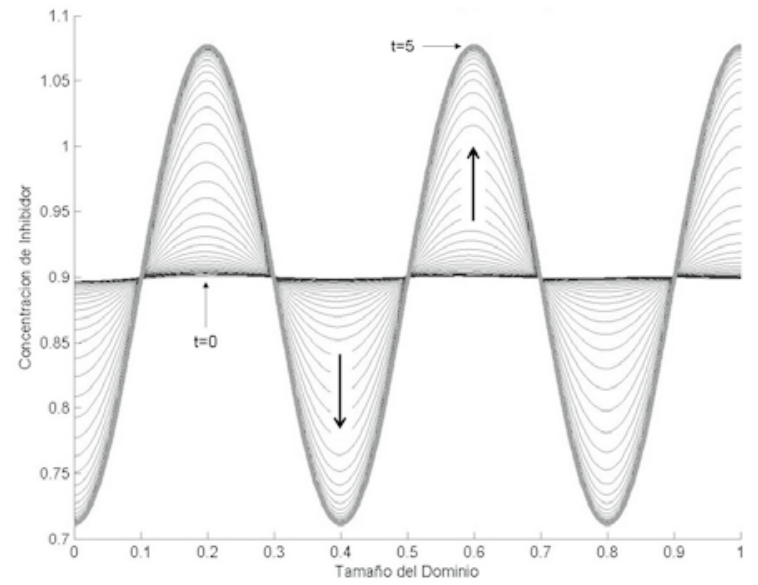

Figura 6. Solución del modelo de Schnakenberg. Concentración de químico inhibidor.

Los resultados muestran el patrón inestable espacial del modelo. Además, la respuesta en desfase de $180^{\circ}$ del activador respecto al inhibidor muestra la acción de este último sobre la producción del primero [9]. El modelo permite confirmar además que pequeñas perturbaciones son determinantes en la formación de patrones espaciales $[1,2,4,7]$. Los resultados mostrados están en completa concordancia con los resultados reportados utilizando para la implementación el método de diferencias finitas $[1,2,8]$.

\section{Modelo de Glucólisis}

El modelo descrito por las ecuaciones (7) fue solucionado en el dominio bidimensional $[0, \pi] \times[0, \pi]$ utilizando los siguientes parámetros: $\mathrm{D}_{\mathrm{u}}=1.0, \mathrm{D}_{\mathrm{v}}=0.0518, \delta=1.75$, $\mathrm{k}=0.05$ [8]. Se realizaron 25.000 iteraciones con un paso de tiempo $\Delta \mathrm{t}=0.1$. Se utilizaron 2.500 elementos cuadriláteros bilineales. Las condiciones de flujo en el contorno se consideran iguales a cero. Las figuras 7 y 8 muestran los resultados obtenidos donde a) es la condición inicial, dada por un nivel de perturbación del $5 \%$ alrededor del estado temporal estable, b) respuesta en $t=750$, c) respuesta en $\mathrm{t}=1.500, \mathrm{y}$ d) respuesta en $\mathrm{t}=2.500$.
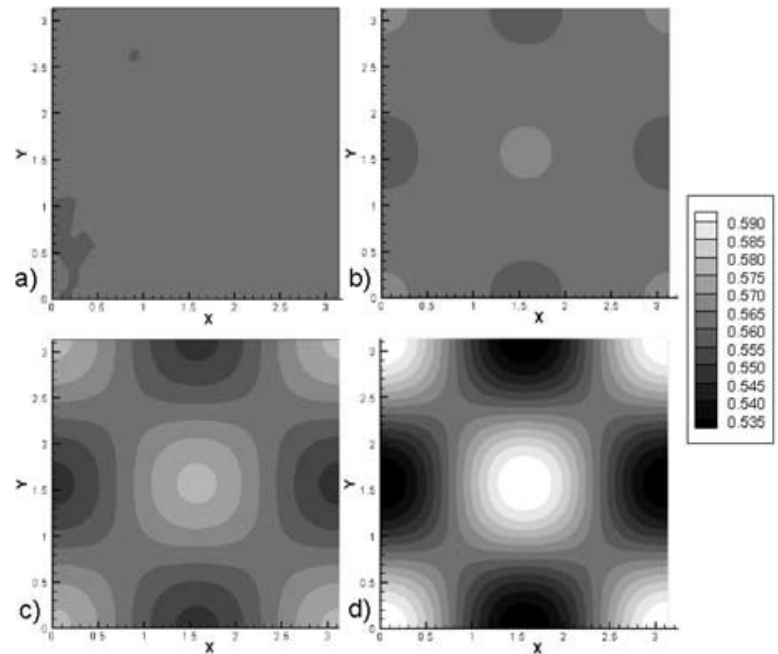

Figura 7. Solución del modelo de glucólisis. Concentración de glucosa.
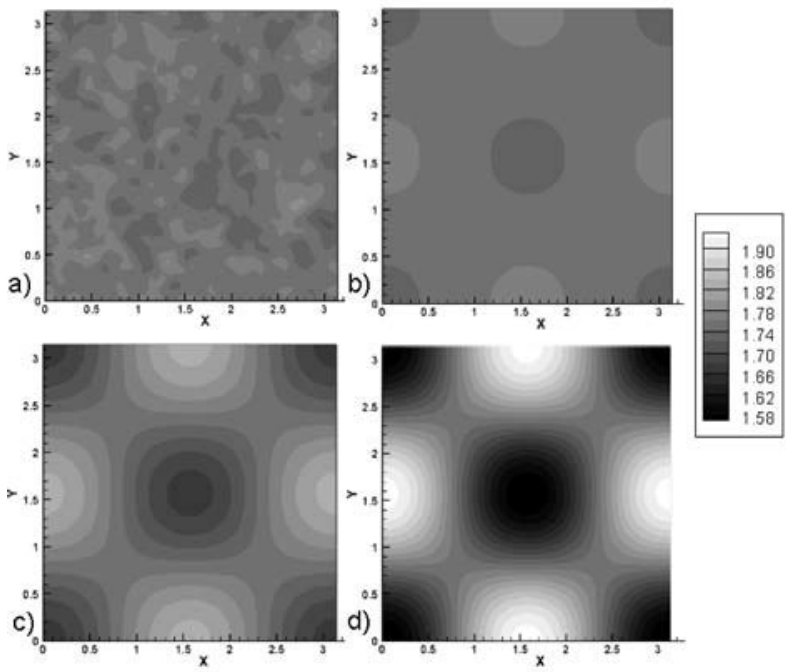

Figura 8. Solución del modelo de glucólisis. Concentración de piruvato. 
Se observa el patrón inestable espacial del modelo y la respuesta en desfase de $180^{\circ}$ entre las dos concentraciones debido a la relación consumo-producción entre los agentes químicos. Las diferencias de intensidad se deben a que el consumo de glucosa es mayor que la producción de piruvato.

Utilizando un dominio bidimensional $[0,5 \pi] \times[0,5 \pi]$ con una malla de 2.500 elementos cuadriláteros bilineales, los parámetros antes usados, y manteniendo las condiciones de la simulación del caso anterior, se obtiene el patrón de puntos mostrado en la figura 9, con a) la condición inicial, dada por un nivel de perturbación del $5 \%$ alrededor del estado temporal estable, b) respuesta en $\mathrm{t}=750$, c) respuesta en $\mathrm{t}=1.500, \mathrm{y} \mathrm{d}$ ) respuesta en $\mathrm{t}=2.500$.
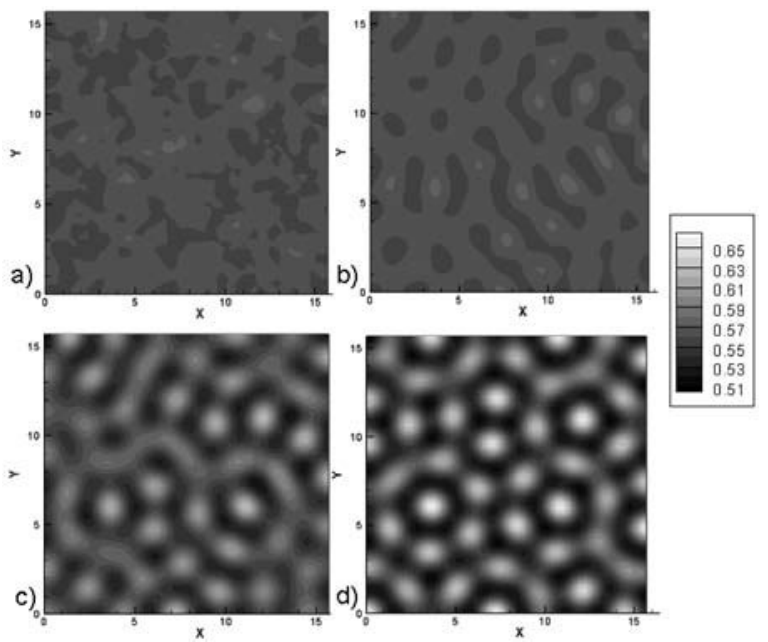

Figura 9. Patrón de puntos en la concentración de glucosa.

\section{Modelo de Quimiotaxis}

El modelo de quimiotaxis con crecimiento celular despreciable, dado por las ecuaciones (8), fue implementado utilizando un dominio bidimensional de dimensiones $[0,10]$ x $[0,10]$ y un mallado de elementos cuadriláteros bilineales de 200 x 20, es decir, un total de 4.000 elementos. Los parámetros utilizados son: $\mathrm{D}_{\mathrm{u}}=0.001, \mathrm{D}_{\mathrm{v}}=0.005$, $\beta=2, \beta=0.1, \delta=0.01$ [8]. La función $\mathrm{g}(\mathrm{n})$ es un escalón unitario que ha sido aproximado por la expresión (23):

$$
g(n)=\gamma \frac{n^{p}}{n^{p}+n_{o}^{p}}
$$

En (24) $n_{0}$ es el valor umbral de densidad celular. Los valores utilizados son: $\mathrm{n}_{0}=0.01, \gamma=0.001$ y $\mathrm{p}=20$ [8]. Las condiciones de flujo en el contorno se consideran iguales a cero. Se realizaron 500 iteraciones con un paso de tiempo $\Delta \mathrm{t}=0.015$. Las figuras 10 y 11 muestran los resultados obtenidos donde a) es la condición inicial, b) respuesta en $t=0.75, c)$ respuesta en $t=3.0, y d$ ) respuesta en $\mathrm{t}=7.5$. La condición inicial en a) está dada por (24):

$$
\begin{aligned}
& n(x, 0)= \begin{cases}n_{i}, & \text { si } x<x_{i} \\
0, & \text { si } x \geq x_{i}\end{cases} \\
& u(x, 0)=u_{i}
\end{aligned}
$$

En (24), los parámetros utilizados son: $\mathrm{n}_{\mathrm{i}}=1.0, \mathrm{x}_{\mathrm{i}}=1.0$, $\mathrm{y} \mathrm{u}_{\mathrm{i}}=0.2[8]$.
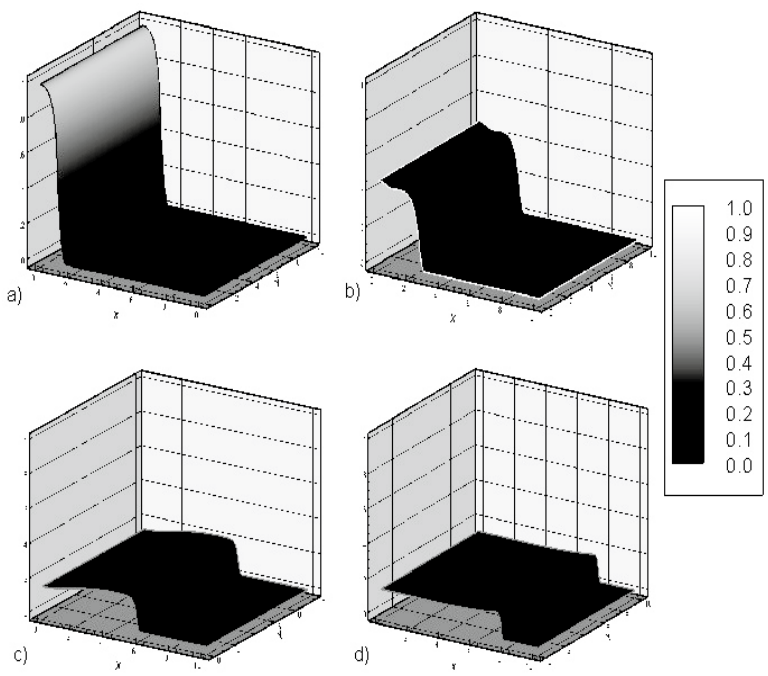

Figura 10. Solución del modelo de quimiotaxis con crecimiento celular nulo. Densidad celular.
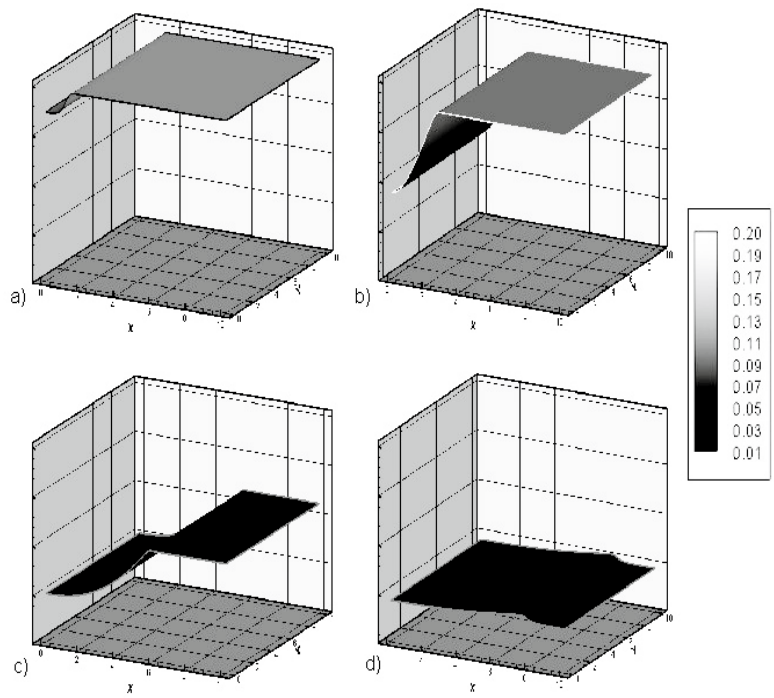

Figura 11. Solución del modelo de quimiotaxis con crecimiento celular nulo. Concentración de quimioatractante. 
Por su parte, el modelo de quimiotaxis que tiene en cuenta el crecimiento y la pérdida celular, descrito por las ecuaciones (9), fue solucionado utilizando un dominio bidimensional de dimensiones $[0,1]$ x $[0,1]$ y el mismo mallado que en el modelo anterior. Los parámetros usados son: $\mathrm{D}_{\mathrm{u}}=0.001, \mathrm{D}_{\mathrm{v}}=0.005, \alpha=2, \beta=0.1, \delta_{\mathrm{u}}=0.02$, $\delta_{\mathrm{v}}=0.05, \gamma=0.001, \mathrm{p}=30, \mathrm{r}=4 \mathrm{n}_{\mathrm{i}}=0.01, \mathrm{x}_{\mathrm{i}}=1.0, \mathrm{y}$ $\mathrm{u}_{\mathrm{i}}=0.2$ [8]. La función $f(u)$ es un escalón unitario que ha sido aproximado por la expresión (25):

$$
f(u)=\frac{u^{p}}{u^{p}+u_{o}^{p}}
$$

En (25), $\mathrm{u}_{0}=0.0195 \mathrm{y} \mathrm{p}=20$ [8]. Las condiciones de flujo en el contorno se consideran iguales a cero. Se realizaron 800 iteraciones con un paso de tiempo $\Delta \mathrm{t}=0.01$. Las figuras 12 y 13 muestran los resultados obtenidos donde a) es la condición inicial, b) es la respuesta en $t=2.5$, c) es la respuesta en $t=5.0$, y d) es la respuesta en $\mathrm{t}=8.0$. a)
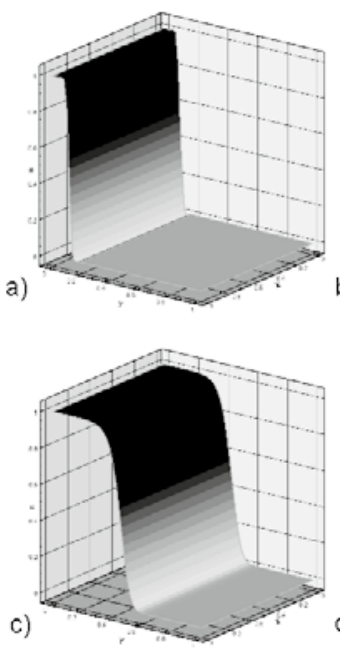
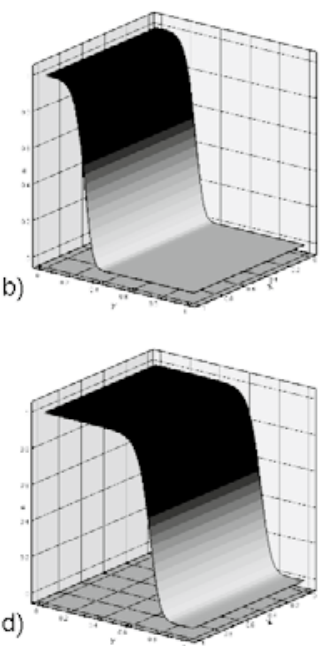

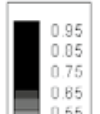

Figura 12. Solución del modelo de quimiotaxis con mitosis celular. Densidad celular.

Las dos implementaciones del modelo de quimiotaxis se ajustan a los resultados obtenidos mediante el método de diferencias finitas reportado en [8]. Se comprueba además la formación del patrón de onda viajera que representa el llamado químico que el quimioactractante ejerce sobre las células.
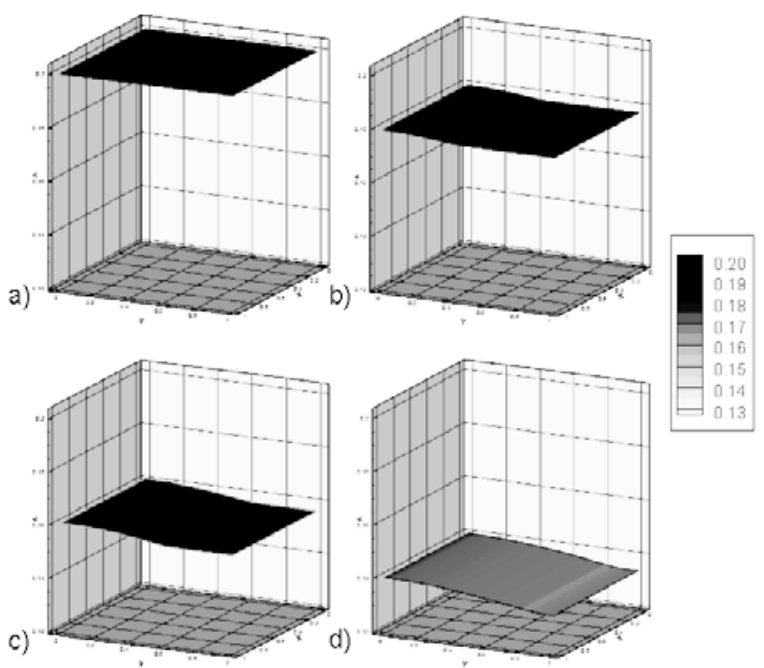

Figura 13. Solución del modelo de quimiotaxis con mitosis celular. Concentración de quimioatractante.

Solucionando el modelo de glucólisis que lleva al resultado de la figura 9 conjuntamente con el modelo de quimiotaxis con mitosis celular que lleva al resultado de la figura 12, se obtiene la formación de tejido producido por las células en migración mostrado en la figura 14. En ella se muestra la aparición del patrón espacio-temporal del modelo de glucólisis a medida que la densidad de células avanza desde el extremo derecho $(x=1)$ hacia el extremo izquierdo $(x=0)$ del dominio. La ecuación que gobierna este resultado está dada por la expresión (26):

$$
\frac{\partial q}{\partial t}=\gamma n v-\beta n q
$$

En (26) $\gamma$ y $\beta$ son parámetros positivos, $q$ es la densidad del tejido en formación, $n$ es la densidad de células del modelo de quimiotaxis y $v$ es la concentración de químico inhibidor del modelo de glucólisis. La solución de (26) se realiza en un dominio bidimensional de dimensiones $[0,1]$ x $[0,1]$ usando 2.500 elementos cuadriláteros bilineales. Los parámetros utilizados son $\gamma=0.05$ y $\beta=0.05$. Se realizaron 25.000 iteraciones con un paso de tiempo $\Delta \mathrm{t}=0.1$. Las condiciones iniciales y de contorno para la variable $q$ son todas iguales a cero. 


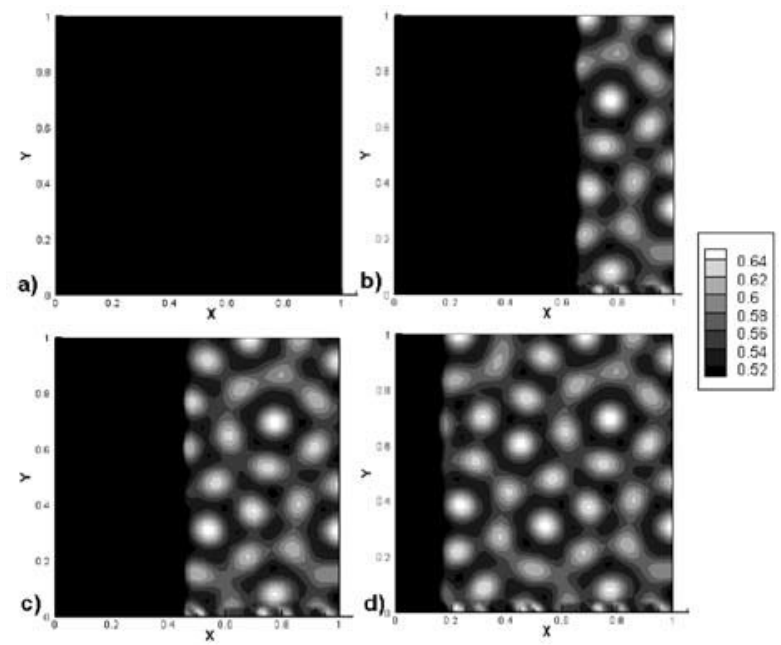

Figura 14. Solución conjunta del modelo de glucólisis y el modelo de quimiotaxis con mitosis celular.

Aunque la ecuación (26) no se encuentra reportada por otros autores, su forma es similar a las ecuaciones de reacción-difusión con el término difusivo igual a cero y su resultado es comparable cualitativamente con resultados reportados por otros trabajos en el área de morfogénesis y recuperación tisular $[2,23]$.

\section{CONCLUSIONES}

Los modelos matemáticos implementados muestran cómo las ecuaciones de reacción-difusión son de utilidad para representar la formación de patrones en sistemas biológicos. Se ha evidenciado la existencia de inestabilidades espaciales debido a pequeñas perturbaciones del estado temporal estable y las variaciones de dichas inestabilidades conforme a los parámetros de cada modelo.

La implementación unidimensional del modelo de Schnakenberg permite visualizar esta formación de patrones espaciales y la evolución en el tiempo de esta formación. Un análisis de los parámetros podría hacerse para identificar características de la formación del patrón, el modo de la onda, y el valor de estado estable [2]. Por ser similares en formulación, la implementación unidimensional de los modelos de Gierer-Meinhardt y Schnakenberg es complementada con la implementación bidimensional del modelo de glucólisis, con lo que se comprueba la validez del método para la solución de problemas de dimensión superior. En el modelo de glucólisis se hace más evidente la variación del patrón espacial inestable conforme a los valores dados a los parámetros del modelo, como bien puede apreciarse en las figuras 7-9. Se observa además el efecto de la dimensión del dominio en la formación del patrón, especialmente en relación con el dominio bidimensional $[0, \pi] \times[0, \pi]$ de las figuras 7 y 8 , y el dominio bidimensional $[0,15] \times[0,15]$ de la figura 9 , hecho que ha sido ampliamente analizado por $[1,17]$. En términos computacionales, estas variaciones del dominio, en conjunto con las dimensiones del mallado utilizado, hacen que la solución requiera de pasos de tiempo ajustados y un número de iteraciones que permita obtener el patrón espacial inestable adecuado, como el caso de los patrones mostrados en las figuras 7-11, visibles sólo después de efectuar 15.000 iteraciones.

El modelo de quimiotaxis, por su parte, se presenta como un ejemplo de fenómenos de movimiento y transporte celular. En particular, los resultados permiten evidenciar la obtención de un patrón espacial que semeja la conformación del frente de onda celular en respuesta a una concentración de quimoatractante. Estos frentes de onda son apreciables en las figuras 10 y 12 . El resultado de este movimiento celular es el marcado consumo de quimioatractante de la figura 11, y el regulado consumo que se aprecia en la figura 13, consecuencia del químico producido por las células añadidas al sistema por el término de crecimiento celular. Es apreciable además la homogeneidad de la formación del patrón a lo largo de todo el dominio, al conservarse siempre su característica ondulatoria.

La implementación de estos modelos utilizando la formulación matemática y el método numérico por elementos finitos presentados ha permitido reproducir los resultados obtenidos por otros métodos reportados en $[1,2,4,8]$. La técnica empleada permite solucionar modelos complejos con menor costo computacional y mejor aproximación numérica, siempre que el dominio, el mallado y las características temporales sean bien especificados. Se espera que la evidencia de los resultados presentados y la técnica de solución empleada sean de utilidad en la formulación e implementación de modelos matemáticos biológicos complejos de crecimiento y desarrollo celular y tisular, de manera similar al resultado mostrado en la figura 14 donde se describe la formación de tejido por parte de las células que migran a lo largo del dominio.

\section{AGRADECIMIENTOS}

Los autores agradecen a la Dirección de Investigación de la Universidad Nacional de Colombia Sede Bogotá por el apoyo económico a los proyectos de investigación en posgrado. Este trabajo hace parte del proyecto de investigación 202010011460 financiado con recursos de la convocatoria DIB Programas de Posgrado 2008 de la Universidad Nacional de Colombia, Bogotá D.C., Colombia. 


\section{REFERENCIAS}

[1] A. Madzvamuse, A.J. Wathen and P.K. Maini. "A moving grid finite element method applied to a model biological patter generator". Journal of Computational Physics. Vol. 190, pp. 478-500. 2003.

[2] D.A. Garzón. "Simulación de procesos de reaccióndifusión: aplicación a la morfogénesis del tejido óseo". Tesis para optar al grado de doctor. Zaragoza, España. 2007.

[3] P.K. Maini. "Mathematical models in morphogenesis". En: Mathematics Inspired by Biology. Springer Berlin- Heidelberg, pp. 151-189. 1999.

[4] P.K. Maini. "Using mathematical models to help understand biological pattern formation". Comptes Rendus Biologies. Vol. 327, pp. 225-234. 2004.

[5] A.M. Turing. "The chemical basis of morphogenesis". Philos. Trans. Roy. Soc. Vol. 237, pp. 37-72. 1952.

[6] K. Page, P.K. Maini and N.A.M. Monk. "Pattern formation in spatially heterogeneous Turing reaction diffusion models". Physics D. Vol. 181, pp. 80-101. 2003.

[7] J.D. Murray. "Mathematical Biology II: Spatial models and biomedical applications". SpringerVerlag, pp. 405-509. 1993.

[8] K.J. Painter. "Chemotaxis as a mechanism for morphogenesis". Thesis to obtain degree of doctor. Oxford University. United Kingdom. 1997.

[9] J.C. Vanegas A., N.S. Landinez P. y D.A. GarzónAlvarado. "Análisis de la inestabilidad de Turing en modelos biológicos". Revista DYNA. Vol. 76 $\mathrm{N}^{\circ} 158$, pp. 123-134. Junio 2009.

[10] Z.C. Wang, W.T. Li and S. Ruan. "Travelling wave fronts in reaction-diffusion systems with spatio-temporal delays". J. Differential Equations. Vol. 222, pp. 185-232. 2006.

[11] P.K. Maini. "Spatial pattern formation in chemical and biological systems". J. Chem. Soc., Faraday Trans. Vol. 93 N $^{\circ}$ 20, pp. 3601-3610. 1997.
[12] H. Meinhardt. "Models of biological pattern formation", pp. 17-19. Academic Press. London. 1982.

[13] J.M. García-Aznar, J.H. Kuiper, M.J. Gómez-Benito, M. Doblaré and J. Richardson. "Computational simulation of fracture healing: Influence of interfragmentary movement on the callus growth". Journal of Biomechanics. Vol. $40 \mathrm{~N}^{\circ}$ 7, pp. 14671476. 2007.

[14] S. Sick, S. Reinker, J. Timmer and T. Schlake. "WNT and DKK determine hair follicle spacing through a reaction-diffusion mechanism". Science. Vol. 314, pp. 1447-1450. 2006.

[15] A. Madzvamuse, A.J. Wathen and P.K. Maini. "A moving grid finite element method applied to a model biological patter generator". Journal of Computational Physics. Vol. 190, pp. 478-500. 2003.

[16] J.D. Murray and G.F. Oster. "Cell traction models for generation pattern and form in morphogenesis". Journal of Mathematical Biology. Vol. 19, pp. 265279. 1984.

[17] A. Madzvamuse. "A numerical approach to the study of spatial pattern formation". Thesis to obtain the degree of doctor. Computing Laboratory. University of Oxford. Oxford, UK. 2000.

[18] D. Ambard and P. Swider. "A predictive mechanobiological model of the bone implant healing". European Journal of Mechanics A/Solids Vol. 25, pp. 927-937. 2006.

[19] T.R. Chandrupatla and A.D. Belegundu. "Introduction to finite elements in engineering". Prentice-Hall, London, pp. 46-100. 1999.

[20] S. Rao. "The finite element method in engineering". Elsevier Science and Technology Books, pp. 1-155. 2004.

[21] E. Oñate, J. Miquel and F. Zárate. "Stabilized solution of the multidimensional advection diffusion - absorption equation using linear finite elements". Computers and Fluids Vol. 36, pp. 92112. 2007 
[22] E. Oñate. "Cálculo de estructuras por el Método de los Elementos Finitos". CIMNE. España, pp. 1-99. 1992.
[23] P. Moreo, J.M. García-Aznar and M. Doblaré. "Bone ingrowth on the surface of endosseous implants. Part 1: Mathematical model". J. Theor. Biol. In Press. 2008. 